\title{
WIRELESS-BASED TECHNOLOGY FOR OPTIMIZING OF OPERATION OF THE AVIATION ENGINE CONTROL SYSTEM
}

\author{
Serhii TOVKACH (i) \\ Automation \& Power Management Department, National Aviation University, Kyiv, Ukraine
}

Received 25 December 2018; accepted 30 September 2020

\begin{abstract}
An approach for improving of efficiency the operation of distributed control system of aviation engine based on wireless technology with high productivity and resolution of wave distributed surface has been presented. It can be applied for development of new principles of correct location the nodes, including the data processing equipment, the intellectual sensors, actuators, repeaters, central units in adaptive control strategies of aviation engine. The optimization method for processing information, using adaptive wavelet filters, as an optimal filter, that minimizes the average square of a common error for organizing the connection between wireless elements in the control systems of aviation gas turbine engine, has been considered, on the theoretical point of view. Also, the wavelets applications in the Wireless Distributed Automatic Control System (WDACS) for aviation engine, the requirements for the construction of its node, supported by the protocol stack, the scheme and the programs with a combination of connections the information exchange between elements have been considered, from the practical point of view.
\end{abstract}

Keywords: aviation, engine, distributed system, wireless, wavelet filter, threshold, subband coding.

\section{Introduction}

The control methods of aviation gas turbine engines currently used in electronic Automatic Control Systems (ACS) are largely similar to those used in the final period of development and operation of systems of hydro mechanical type (in the 70s and 80s). They were improved in the direction of a large adaptation of control to operating conditions, integration with the flight control systems. New solutions in the field of adaptive and integrated control, control in the case of failures and damages of the engine ACS and others are being intensively developed and, obviously, more fully will be increasingly used in the near future.

Important and considered in the development of ACS Gas Turbine Engine (GTE) are the issues to ensure their operation with minimal maintenance costs. The possibility of operation according to the technical condition is characteristic of the best modern control systems.

Prospects for the development of ACS GTE associated with the transition to the construction of digital electronic systems of a distributed structure based on the use of faultstability SMART-subsystems (intellectual sensors and actuators), coordination of the subsystems with a central redundant processor, integrated control, high-performance communications with other onboard systems (Figure 1).
Such construction of control systems will significantly increase reliability, reduce the mass of equipment (by 30 to $40 \%$ ), improve the quality of control and, as a result, improve engine performance, reduce production and operation costs (up to 50 and 60\%) (Gurevich, 2010).

It should be noted that the work on the creation of distributed control systems in foreign research centers (Glenn Research Center (NASA), PW, GE, RR, Snecma, BAE Systems, Hamilton Sandstrand, Honeywall) is actively conducted (Gurevich, 2010; EUROCAE, 2020). The basis for constructing such control systems (Oliveira et al., 2017) are wireless intellectual speed sensors (WIS $n_{s}$ and WIS $n_{k}$ ), wireless intellectual temperature and pressure sensors at the input to the engine (WIS $T_{i n}^{*}$ and WIS $p_{\text {in }}^{*}$ in), wireless intellectual pressure sensors behind the compressor (WIS $p_{c}^{*}$ ), wireless intellectual temperature of the gases sensor behind the turbine (WIS $T_{t}^{*}$ ); wireless intellectual actuators of fuel consumption control (WIA $G_{T}$ ), wireless intellectual actuators the guiding devices of the compressor (WIA GD), wireless intellectual actuators of nozzles (WIA N).

An essential feature of the GTE as a control object is that its number of regulated parameters exceeds the number of regulatory factors (Calvo et al., 2020), which largely determines the specifics of the construction of the ACS gas turbine engine. The choice of the combination of regulated

${ }^{*}$ Corresponding author. E-mail: ss.tovkach@gmail.com 


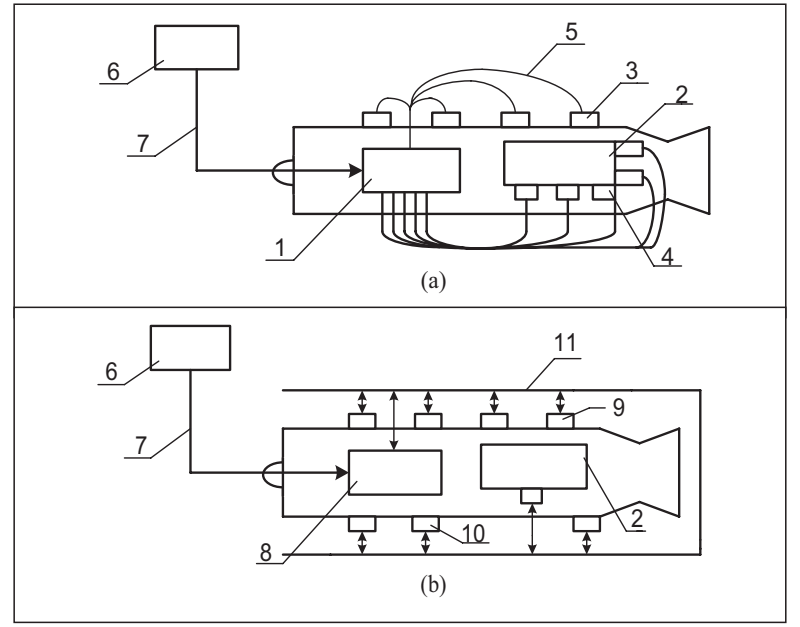

Figure 1. Structural construction of ACS gas turbine engine with centralized (a) and distributed (b) structure: 1 - electronic controller FADEC; 2 - fuel system; 3 - sensors; 4 - executive mechanisms; 5 - analog lines; 6 - ACS of aircraft; 7 - MIECH (Multiplex Information Exchange Channel) of aircraft; 8 - the central processor of the ACS gas turbine engine; 9 - intellectual sensors; 10 - intellectual actuators; 11 - MIECH of GTE

parameters and the control factors of the engine depends on the purpose of the engine, the requirements for its characteristics, the scheme of construction, the number of regulated elements, etc. These combinations are set in the form of control programs (Tech Briefs, 2020), which are the dependence of the regulation parameters (or regulatory factors) on external conditions $\left(T_{i n}^{*}\right)$, regulating factors, and sometimes other regulatory parameters.

The goal of this research is to define methods of construction and control programs that should allow optimizing the correct operation of aviation engine control system, providing the strength of its design based on wireless information exchange.

Such an approach implies the use of the principle of automatic control systems of aviation gas turbine engine (Gurevich, 2010), wireless technology intra-aircraft wireless data bus for essential critical applications (EUROCAE, 2020), methods for control and diagnostics of aircraft gas turbine engine (Antonov et al., 2013).

According to the goal, it is necessary to investigate the influence of failures (Chilupuri, 2018), during the data processing between wireless nodes and Onboard Central Processor Unit (OCPU), leading to distortion the control actions generated in the OCPU (Bazhenov \& Kalenov, 2017), implementing recursive and non-recursive algorithms, on the quality of ACS control processes (Securaplane Technology Inc., 2019), and propose the effectiveness method or control program using a certain combination of algorithms in digital WDACS (Dias et al., 2018) to ensure reliance to failures.

\section{Optimizing method of engine control system operation}

The specified control algorithms (including digital filtering algorithms) for power plants are implemented by the digital correction filters (programmatically on the OCPU or as a specialized digital device), which for most cases can be built either by recursive or by non-recursive schemes.

Let analyze the systems that minimize the average square of the error (difference) between the total signal at the input of the system and the useful signal at its input, using the s-plane (Sternberg, 2019). According to Figure 2, $S_{X}(s)$ is the spectral density for the incoming random signal $X(t), S_{N}(s)$ is the spectral density of the input noise $N(t), S_{Y}(s)$ and $S_{M}(s)$ is the spectral density of the output signal $Y(t)$ and the output noise $M(t)$. The assumption, that, the input noise is white noise, does not lead to a significant simplification of the optimization procedure, so this assumption will not be accepted.

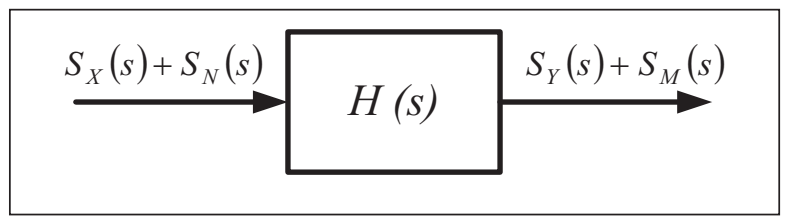

Figure 2. Spectral density of signals and noise at the input and output of the control system

An error in the signal component, due to the system itself, is defined as $E(t)=X(t)-Y(t)$.

The Laplace transform of this error has the form

$$
\begin{aligned}
& F_{E}(s)=F_{X}(s)-F_{Y}(s)=F_{X}(s)-H(s) \times \\
& F_{X}(s)=F_{X}(s)[1-H(s)] .
\end{aligned}
$$

Accordingly, $[1-H(s)]$ is a transmitting function, that binds the signal error to the input signal, and the value of the average square of this error is equal to

$$
\bar{E}^{2}=(1 / 2 \pi j) \int_{-j \infty}^{j \infty} S_{X}(s)[1-H(s)][1-H(-s)] d s .
$$

The value of the average square of the noise at the output of the system is determined from the expression

$$
\bar{M}^{2}=(1 / 2 \pi j) \int_{-j \infty}^{j \infty} S_{N}(s) H(s) H(-s) d s .
$$

Due to the statistical independence of the signal and noise, the total value of the average square of the error is equal $\bar{E}^{2}+\bar{M}^{2}$ and is determined from the ratio

$$
\begin{aligned}
& \bar{E}^{2}+\bar{M}^{2}=(1 / 2 \pi j) \int_{-j \infty}^{j \infty}\left\{S_{X}(s)[1-H(s)] \times\right. \\
& \left.[1-H(-s)]+S_{N}(s) H(s) H(-s)\right\} d s .
\end{aligned}
$$

Now, it is need to define the form of a function $H(s)$ that minimizes the expression (4).

If the system was not bound by its mandatory casualism (Wiener, 2014), the procedure for determining of the optimal type of function would be simple. To show this, it can be regroup the addons of the subintegral expression (4) and get it

$$
\begin{aligned}
& \bar{E}^{2}+\bar{M}^{2}=(1 / 2 \pi j) \int_{-j \infty}^{j \infty}\left\{\left[S_{X}(s)+S_{N}(s)\right] H(s) \times\right. \\
& \left.H(-s)-S_{X}(s) H(s)-S_{X}(s) H(-s)+S_{X}(s)\right\} d s .
\end{aligned}
$$


As well as $\left[S_{X}(s)+S_{N}(s)\right]$ is spectral density, it must have the property of symmetry (parity) and can be represented in the form of two conjugates, one of which has poles and zeros in the left half-plane, and the second one is analogous poles and zeros in the right half-plane, that is, it is possible write down

$$
S_{X}(s)+S_{N}(s)=F_{i}(s) F_{i}(-s) .
$$

Substituting of expression in (5) and re-grouping of terms of the subintegral expression, can be obtain

$$
\begin{aligned}
& \bar{E}^{2}+\bar{M}^{2}=(1 / 2 \pi j) \int_{-j \infty}^{j \infty}\left\{\left[F_{i}(s) H(s)-S_{X}(s) / F_{i}(-s)\right] \times\right. \\
& {\left[F_{i}(-s) H(-s)-S_{X}(s) / F_{i}(s)\right]+S_{X}(s) \times} \\
& \left.S_{N}(s) / F_{i}(s) F_{i}(-s)\right\} d s .
\end{aligned}
$$

Now, it can be seen, that the last term in the subintegral expression (7) does not include $H(s)$. As a consequence, taking into account that the product, which is the first plural of the integral expression, can not be negative, a minimum of the sum $\bar{E}^{2}+\bar{M}^{2}$ will occur with the equality of its factors equal to zero. This means that the optimal transfer function is equal to

$$
H(s)=S_{X}(s) /\left[F_{i}(s) F_{i}(-s)\right]=S_{X}(s) /\left[S_{X}(s)+S_{N}(s)\right] \text {. }
$$

The validity of the obtained result would seem obvious, except that the function (8) is symmetric in the splane, and therefore can not be an analytical representation of the casual system.

So, as the transfer function, which is determined by the relation (8), does not describe the casual system, the procedure involves the use of the poles and zeros of the left half-plane of expression (8) for the description of the casual system. An analogy with the "throwback" could give of the function part $s\left(t_{0}-t\right)$ for $t<0$ a coherent filter (Vamvoudakis, 2016). Unfortunately, this task is not simple enough, since in this case, the random process $X(t)+N(t)$ at the system input is not a white noise. If this process was white noise, then its correlation function would be a $\delta$-function and, accordingly, all future values of the input process would be uncorrelated with its present and past values. Thus, a system that, can not respond to future inputs (that is, a casual system), will not reject the use of any information that, can only lead to an improved signal estimation. Therefore, it seems that the first step in determining the structure of the casual system should be to convert the amount of signal and noise into white noise, which requires the use of the "purposive" filter.

From (6) it is obvious that, if the transfer function of the filter $H_{1}(s)$ is equal to

$$
H_{1}(s)=1 / F_{i}(s),
$$

then the process at its output is a white noise, since fair equality

$$
\begin{aligned}
& {\left[S_{X}(s)+S_{N}(s)\right] H_{1}(s) H_{1}(-s)=\left[S_{X}(s)+S_{N}(s)\right] /} \\
& F_{i}(s) F_{i}(-s)=1 .
\end{aligned}
$$

In addition, $H_{1}(s)$ describes the casual system, since by definition $F_{1}(s)$ has poles and zeros only in the left half-plane. Thus, $H_{1}(s)$ is the transfer function of the purposive filter for the sum of the input signal and the input noise.

Once more, let analyze the term $\left\{F_{i}(s) H(s)-\left[S_{X}(s) / F_{i}(-s)\right]\right\}$ in the subintegral expression (7), which can be considered to be zero. The presence of poles, located in the right half-plane, is due to its second member, which in turn can represent (by decomposition into simple fractions) in the form of the sum of two terms, one of which has poles only in the left half-plane, and the other, only in the right half-plane. Then the whole expression for this plural form will look

$$
\begin{aligned}
& F_{i}(s) H(s)-\left[S_{X}(s) / F_{i}(-s)\right]= \\
& F_{i}(s) H(s)-\left[S_{X}(s) / F_{i}(-s)\right]_{L}-\left[S_{X}(s) / F_{i}(-s)\right]_{R},
\end{aligned}
$$

where the indexes " $\mathrm{L}$ " and " $\mathrm{R}$ " are mean respectively the poles of the left and only the right half-plane. Now it is clear that for $H(s)$ that, descript a casual system, the analyzed singing multiplier can not be considered equal to zero, and the smallest value that, it can have, can be obtained by zeroing of difference between the first two members in the right-hand side (10): $F_{i}(s) H(s)-\left[S_{X}(s) / F_{i}(-s)\right]_{L}=0$, or

$$
H(s)=\left[1 / F_{i}(s)\right]\left[S_{X}(s) / F_{i}(-s)\right]_{L} .
$$

It should be noticed, that the first coefficient in (11) is the transmitting function $H_{1}(s)$ of the purposive filter. Thus, the best which, can be done for minimizing the average square of the general error, is to get rid of noncasual components (that is, the components that, describe non-casual systems) in the second co-multiplier in (12).

An optimal filter (Calvo et al., 2020) that, minimizes the average square of a common error can be called an adaptive wavelet filter, which can be viewed as two sequentially connected devices (Figure 3 ). The first of which is a subband coding filter with a transfer function $H_{1}(s)$, and the second with a transfer function $H_{2}(s)$ actually a wavelet filter. Often functions $H_{1}(s)$ and $H_{2}(s)$ have common co-multipliers, after the mutual reduction of which, the general transfer function acquires a simpler form than, it was possible to expect, and becomes more simple in realization than, each of its output multipliers $H_{1}(s)$ and

As an example of an optimal filter (Cunha et al., 2016), can be considered the case (Bjorkbom et al., 2011), when the input useful signal $X(t)$ and the input noise $N(t)$ have

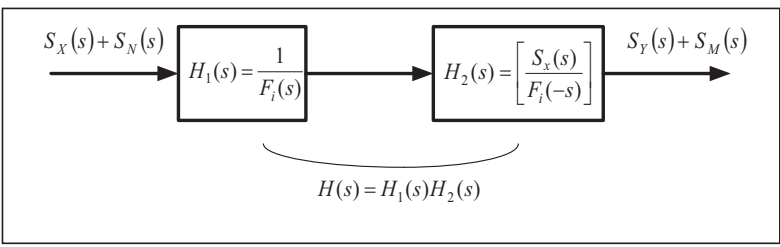

Figure 3. Optimal filter for system operation 
the corresponding spectral densities $S_{x}(s)=-1 /\left(s^{2}-1\right)$ and $S_{N}(s)=-1 /\left(s^{2}-4\right)$ then can be obtained

$$
\begin{aligned}
& F_{i}(s) F_{i}(-s)=S_{X}(s)+S_{N}(s)=\left[-1 /\left(s^{2}-1\right)\right]-\left[1 /\left(s^{2}-4\right)\right]= \\
& -\left(2 s^{2}-5\right) /\left(s^{2}-1\right)\left(s^{2}-4\right),
\end{aligned}
$$

where follows

$$
F_{i}(s)=\sqrt{2}(s+\sqrt{2,5}) /(s+1)(s+2) .
$$

Therefore, the transfer function of the subband filter is equal to

$$
H_{1}(s)=1 / F_{i}(s)=(s+1)(s+2) / \sqrt{2}(s+\sqrt{2.5}) .
$$

The transfer function $\mathrm{H}_{2}(s)$ of the second filter (Figure 2 ) is easily determined from the ratio

$$
\begin{aligned}
& S_{X}(s) / F(-s)=\left[-1 /\left(s^{2}-1\right)\right](-s+1)(-s+2) / \\
& \sqrt{2}(-s+\sqrt{2.5})=(s-2) / \sqrt{2}(s+1)(s-\sqrt{2.5}),
\end{aligned}
$$

which can be represented in the form of a schedule for simple fractions

$$
S_{X}(s) / F_{i}(-s)=[0.822 /(s+1)]-[0.115 /(s-\sqrt{2.5})] \text {. }
$$

Accordingly, the transfer function $H_{2}(s)$ is equal to

$$
H_{2}(s)=\left[S_{X}(s) / F_{i}(-s)\right]_{L}=0.822 /(s+1) .
$$

Then the transfer function of the optimal filter is generally defined as

$$
\begin{aligned}
& H(s)=H_{1}(s) H_{2}(s)=[(s+1)(s+2) / \\
& \sqrt{2}(s+\sqrt{2.5})][0.822 /(s+1)]=0.582(s+2) /(s+\sqrt{2.5}) .
\end{aligned}
$$

Now, it remains, to estimate the characteristics (Bradley et al., 2020) of the optimal filter, that is, to determine the true value of the minimum average square of the complete error. The solution of this problem is greatly simplified, if for the optimal system of this filter count the fact of the non-correlation of the final error and of the process at the output of the system. If this statement were not fair, then it would be possible to continue to perform linear operations over the output signal and receive even less errors. Thus, the minimum value of the average square of the error is simply the difference between the values of the average square of the input signal component and the total process at the filter output:

$$
\begin{aligned}
& \left(\bar{E}^{2}+\bar{M}^{2}\right)_{\min }=(1 / 2 \pi j) \times \\
& \int_{-j \infty}^{j \infty} S_{X}(s) d s-(1 / 2 \pi j) \int_{-j \infty}^{j \infty}\left[S_{X}(s)+S_{N}(s)\right] H(s) H(-s) d s,
\end{aligned}
$$

where $H(s)$ is defined in accordance with (12).

The given result can be used to determine the minimum value of the mean squared error in relation to the adaptive wavelet filter, which is described by the transfer function of the form (19). The first integral in (20) is easy to calculate or using Table 1 , or by summing the remains. As a result can be received

$$
(1 / 2 \pi j) \int_{-j \infty}^{j \infty} S_{X}(s) d s=(1 / 2 \pi j) \int_{-j \infty}^{j \infty}\left[-1 /\left(s^{2}-1\right)\right] d s=0.5 \text {. }
$$

Similarly, the second integral in (20) is calculated in 22 .

$$
\begin{aligned}
& (1 / 2 \pi \mathrm{j}) \int_{-j \infty}^{j \infty}\left[S_{X}(s)+S_{N}(s)\right] H(s) H(-s) d s= \\
& (1 / 2 \pi j) \int_{-j \infty}^{j \infty}\left[-2(0.582)^{2} /\left(s^{2}-1\right)\right] d s=0.339 .
\end{aligned}
$$

Then the minimum value of the average square of the error is equal to

$$
\left(\bar{E}^{2}+\bar{M}^{2}\right)_{\min }=0.5-0.339=0.161 .
$$

It is interesting to compare this value with the lack of a filter. In this case, there is no signal error, and the average square of the complete error is equal to the average square of the noise

$$
\bar{E}^{2}+\bar{M}^{2}=\bar{N}^{2}=(1 / 2 \pi j) \int_{-j \infty}^{j \infty}\left[-1 /\left(s^{2}-4\right)\right] d s=0.25 \text {. }
$$

It is clear from this that, the use of the filter results in a significant reduction in the total error (Hassan, 2017), and the degree of this decrease would be even more pronounced with a wider band of input noise.

Table 1. Integrals (the relationship between the mean square of a random process and the spectral density)

\begin{tabular}{|l|l|}
\hline \multicolumn{1}{|c|}{ Expression } & \multicolumn{1}{c|}{ Explanation } \\
\hline$I_{n}=(1 / 2 \pi j) \int_{-j \infty}^{j \infty} \frac{\tilde{n}(s) \tilde{n}(-s)}{d(s) d(-s)} d s$ & the mean square of a random process on a complex plane \\
\hline$I_{1}=\frac{c_{0}^{2}}{2 d_{0} d_{1}}$ & the mean square of a random process on a complex plane for $n=1$ \\
\hline$I_{2}=\frac{c_{1}^{2} d_{0}+c_{0}^{2} d_{2}}{2 d_{0} d_{1} d_{2}}$ & the mean square of a random process on a complex plane for $n=2$ \\
\hline$I_{3}=\frac{c_{2}^{2} d_{0} d_{1}+\left(c_{1}^{2}-c_{0} c 2\right) d_{0} d_{3}+c_{0}^{2} d_{2} d_{3}}{2 d_{0} d_{3}\left(d_{1} d_{2}-d_{0} d_{3}\right)}$ & the mean square of a random process on a complex plane for $n=3$ \\
\hline
\end{tabular}




\section{Engine system wireless-based technology operation}

Node interaction. The block diagram of adaptive wavelet filtration of the wave distribution for wireless nodes can be considered in Figure 4 .

The signal filtering is performed as follows:

1. collecting sample signal data, decomposition of the signal by the base wavelet functions ("purposive" filter);

2. calculation of the function thresholds for each decay band depending on the statistical properties of the signal according to the variance by the formula

$T_{\text {adapt }, j}=\sigma_{j} \sqrt{2 \lg n}$,

where $\sigma_{j}^{2}=D x_{j}$ is the variance of wavelet coefficients in the $j$ band;

3. Threshold processing of coefficients (wavelet filtering);

4. Iinverse wavelet transform.

The result of the signal filtering based on mathematical technique, which has been considered for optimizing the operation of aviation engine control system, can be performed by the input experimental signal from wireless sensors CA-191 the D-27 engine of the electronic control system $27 \mathrm{M}$.

Full visualization of procedures for compression and removal of noise allows and qualitatively processes the signal. Results can be saved using the capabilities of the usual Windows-name (Figure 5): original with high frequency signal on transition mode and denoised useful signal.

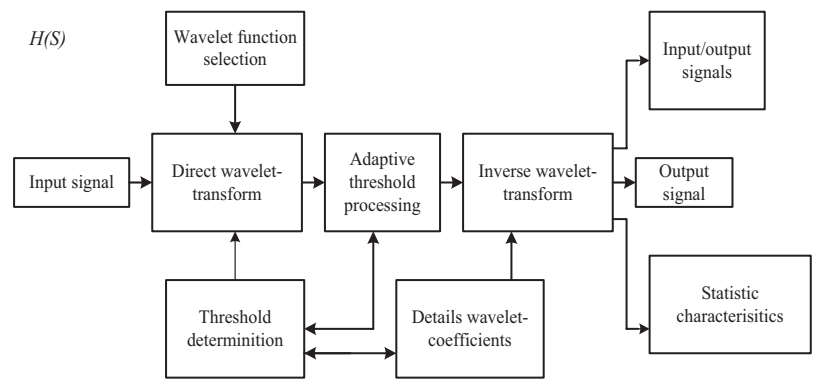

Figure 4. Block diagram of adaptive wavelet signal processing

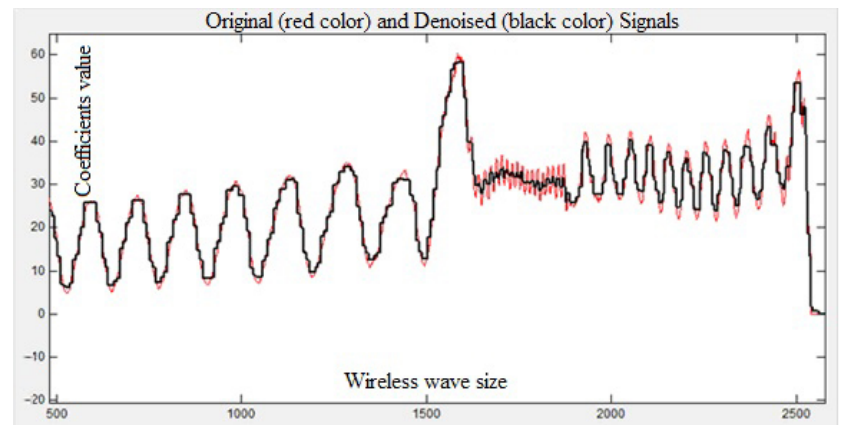

Figure 5. Result of the signal filtering
Technique control programs. One of the possible combinations of engine-type control programs (Bieniek, 2011) based on wireless technology, which provide control over steady and transition operating modes in all operating conditions, using the optimal system functioning can be defined:

1. Regulation at maximum engine operation (fuel consumption control $G_{T}$, compressor control).

2. Regulation of the gas turbine engine on throttle modes.

3. Regulation programs of low gas mode.

4. Control on acceleration and gas release modes (effect of heat removal and heating of the structure).

5. Control on the start mode.

6. Gas turbine engine control programs on forced operation modes (control on full forced mode, regulation on throttle forced modes, regulation on transition conditions, when the degree of forcing changes, engine protection from compressor surge).

The complex control algorithms can be implemented in OCPU, which in particular, include algorithms for reducing interference effects, i.e. digital filtering algorithms. To specify a filter, you need to know the values of the coefficients, the count of the input sequence and the initial values (Ashok et al., 2016).

The diagram of the distributed structure of the WACS (Wireless Automatic Control System) GTE with the use of wireless sensor networks can be seen in Tovkach (2020), which will reduce the number of radial lines due to the transition of multiplex information exchange channels, simplify the localization of failures and restructuring of the structure; reduce the likelihood of destruction of the system and, thus, increase its survivability (Tanenbaum, 2016).

\section{Conclusions}

The analytical review and the analysis of data from other sources can be concluded that the efficient functioning of the control system of aviation engine depends on structural construction of ACS: centralized or distributed with SMART-subsystems, according to the proposed wireless scaling technology due to the standard IEEE 802.15.4.

A distinctive feature of the design and correct operation of the ACS is the number of regulated parameters and the number of regulatory factors, which is the set of proposed control programs and depends on the requirements for engine characteristics.

The method of wave distribution between wireless nodes of ACS based on digital filtering algorithm is adaptive wavelet filtering with subband coding, integral calculations and summing the remains to determine the minimum value of the mean squared error has been considered. It helps to better understand the creation of efficiency path interaction between nodes.

The structure diagram and result of signal filtering for adaptive information processing as a wavelet filtration, wavelet estimation with usage of different threshold 
processing is given and on their fundamentals study the distribute ACS gas turbine engine has been modified.

In this way, the proposed approach for information processing between the elements of the electronic WACS can be used, when constructing a system for diagnosing of technical state of the GTE and a control system with a flexible, easily variable structure. The implementation of wireless technologies in the ACS GTE will reduce the mass and size of nodes by reducing the number of connectors and cables, increasing the reliability of the ACS.

\section{References}

Antonov, A. O., Trofimenko, R. A., \& Yatsko, L. L. (2013). Modern methods of control and diagnostics of aircraft gas turbine engine. Aerospace Technic and Technology, 10, 141-145 (in Russian). http://nbuv.gov.ua/UJRN/aktit_2013_10_27

Ashok, B., Ashok, S. D., \& Kumar, C. R. (2016). A review on control system architecture of a SI engine management system. Annual Reviews in Control, 41, 94-118. https://doi.org/10.1016/j.arcontrol.2016.04.005

Bazhenov, Y. V., \& Kalenov, V. P. (2017). The forecasting of the residual resource of electronic engine control system. The Russian Automobile and Highway Industry Journal, 2(54), 52-59. https://doi.org/10.26518/2071-7296-2017-2(54)-52-59

Bieniek, A. (2011). Advanced nonroad diesel engine control system. Machine Design, 3(3), 167-172.

Bjorkbom, M., Nethi, Sh., \& Eriksson, L. M. (2011). Wireless control system design and co-simulation. Control Engineering Practise, 19(9), 1075-1086. https://doi.org/10.1016/j.conengprac.2011.05.012

Bradley, N., Karipott, S. S., Wang, Y, \& Ong, K. G. (2020). Wireless technologies for implantable devices. Sensors, 20(16), 4604. https://doi.org/10.3390/s20164604

Calvo, I., Abrahams, S., Barambones, O., \& Quesada, J. (2020). A comparison of wired and wireless technologies for control applications. In Proceedings of the XXXIX Journadas de Automatica, Badajoz (pp. 538-545).

https://doi.org/10.17979/spudc.9788497497565.0538

Chilupuri, A. (2018). Automation in wireless control system: a small review study of automation of water motor using Zig-
Bee. International Journal for Research in Applied Science \& Engineering Technology, 6(4), 512-514.

https://doi.org/10.22214/ijraset.2018.4089

Cunha, J. P. P., Cardeira, C., Batista, N. C., \& Melicio, R. (2016). Wireless technologies for controlling a traffic lights prototype [Conference presentation]. IEEE International Power Electronics and Motion Control Conference, Vama, Bulgaria (pp. 858-863). https://doi.org/10.1109/EPEPEMC.2016.7752108

Dias, B. M. de A., Lagana, A. M. A., J. F. Justo, Yoshioka, L. R., Santos, M. M. D., \& Gu, Z. (2018). Model-based development of an engine control module for a spark ignition engine. IEEE Access, 6, 1-12. https://doi.org/10.1109/ACCESS.2018.2870061

EUROCAE. (2020). Standarts for future aviation. https://www. eurocae.net/

Gurevich, O. S. (2010). Automatic control systems of aviation gas turbine engine (264 p.). Toru Press (in Russian).

Hassan, S. M. (2017). Application of wireless technology for control: a wireless HART perspective. Procedia Computer Science, 105, 240-247. https://doi.org/10.1016/j.procs.2017.01.217

Oliveira, J., Coelho, A., Stefenon, S., \& Yamaguchi, Cr. (2017). Stochastic approach is Markov chain applied to the analysis and project of the information systems oriented to object. International Journal of Development Research, 07(06), 13139-13143.

Securaplane Technology Inc. (2019). Wireless technology intraaircraft wireless data bus for essential and critical applications. https://www.securaplane.com/

Sternberg, S. (2019). A mathematical companion to quantum mechanics (336 p.). Dover Publications.

Tanenbaum, A. (2016). Distributed systems: principles and paradigms (702 p.). CreateSpace Independent Publishing Platform.

Tech Briefs. (2020). Radio-frequency wireless flight-control system. https://www.techbriefs.com/

Tovkach, S. S. (2020). The Liebman process for distribution of the information flows of the engine automatic control system. Journal of Nano- and Electronic Physics, 1(12), 01003(1)01003(5). https://doi.org/10.21272/jnep.12(1).01003

Vamvoudakis, K. (2016). Control of complex systems: theory and applications (762 p.). Butterworth-Heinemann.

Wiener, N. (2014). The Fourier integral and certain of its applications (216 p.). Martino Fine Books. 